\title{
Myxomycetes in Turkey - a checklist
}

\section{Ertuğrul Sesli 1*, Ilgaz Akata ${ }^{2}$, Teodor T. Denchev ${ }^{3} \&$ Cvetomir M. Denchev ${ }^{3}$}

\author{
${ }^{1}$ Fatih Faculty of Education, Karadeniz Technical University, 61335 Sogutlu Trabzon, Turkey \\ ${ }^{2}$ Department of Biology, Faculty of Science, Ankara University, 06100 Ankara, Turkey \\ ${ }^{3}$ Institute of Biodiversity and Ecosystem Research, Bulgarian Academy of Sciences, 2 Gagarin St., 1113 \\ Sofia, Bulgaria
}

Received 3 February 2016 / Accepted 10 February 2016 / Published 13 February 2016

Sesli, E., Akata, I., Denchev, T.T. \& Denchev, C.M. 2016. Myxomycetes in Turkey - a checklist. - Mycobiota 6: 1-20. doi: 10.12664/mycobiota.2016.06.01

\begin{abstract}
This checklist contains 252 species of myxomycetes recognized as occurring in Turkey. The names are arranged alphabetically. For each species, references are cited. An index of synonyms, based on literature records from Turkey, is appended.
\end{abstract}

Key words: fungal diversity, myxomycetes, Turkey

\section{Introduction}

This paper presents our current knowledge about the species diversity of myxomycetes (a group of free-living, unicellular or plasmodial phagotrophic eukaryotes) in Turkey. Turkey is the meeting ground of three phytogeographical regions-Euro-Siberian, Mediterranean, and Irano-Turanian (Davis et al. 1971). The Euro-Siberian Region extends along most of north Anatolia also occurs as a narrow strip along the Black Sea coast of European Turkey. The relatively humid climate is reflected in the predominantly mesophytic vegetation. The Mediterranean Region covers all areas bordering the Mediterranean Sea and reaches its eastern limits in the Anti-Taurus and Amanus. The landscape of this region is dominated by sclerophyllous vegetation. The Irano-Turanian Region is the largest phytogeographical region of Turkey and is confined to Central and East Anatolia. The extraordinary phytogeographical conditions of Anatolia have produced considerable species diversity. The Irano-Turanian flora in Turkey is closely related to that of Transcaucasia, NW and W Iran, and N Iraq (Davis 1965-1988). While there are 12500 gymnosperm and angiosperm plant species in the entire continent of Europe, it is known that there is a similar

\footnotetext{
*Corresponding author: e-mail: ertugrulsesli@yahoo.com
} 
number (about 11,000) in Anatolia alone, with some one-third of them endemic to Turkey (Ministry of Environment and Forestry 2007). This is a prerequisite for a high species richness of myxomycetes.

The first records of myxomycetes from Turkey were published by Lohwag (1957, 1964), Härkonen \& Uotila (1983), Gücin \& Öner (1986), and Härkonen (1987). In the middle '90s of the last century a number of Turkish researchers began to study intensively myxomycetes. Checklists of the Turkish myxomycetes have been compiled by Ergül \& Dülger (2000), Sesli \& Denchev (2005, 2008, 2014), and Dülger (2007).

In the main list of myxomycetes that follows, taxa are given in alphabetical order. The generic and species treatment follows the Eumycetozoa database (Lado 2016). Because some species have been published under different names, a list of synonyms is separately given with references to the correct names used in the main list. A short list of excluded records, providing reasons for their exclusion, is also appended.

\section{List of myxomycetes}

PERCOLOZOA

Acrasida

Guttulinaceae

Pocheina rosea (Cienk.) A.R. Loebl. \& Tappan (Gücin et al. 1996a)

\section{MYCETOZOA}

Protostelea

Protostelida

Ceratiomyxaceae

Ceratiomyxa fruticulosa (O.F. Müll.) T. Macbr. (Härkönen 1988; Ergül \& Dülger 2000d; Ergül et al. 2005a, b; Ocak \& Hasenekoğlu 2005; Yağız \& Afyon 2005, 2006a; Demirel et al. 2006; Oran et al. 2006; Baba \& Tamer 2008a; Ergül \& Akgül 2011; Baba et al. 2013b, 2015; Eroğlu \& Kaşik 2013a; Baba \& Zümre 2015; Oskay \& Tüzün 2015)

\section{Myxogastria}

\section{Echinostellida}

Clastodermataceae

Clastoderma debaryanum A. Blytt (Ergül \& Dülger 2000d; Ergül et al. 2005b; Ocak \& Hasenekoğlu 2005; Oran et al. 2006; Baba et al. 2013b; Eroğlu \& Kaşik 2013a), C. pachypus Nann.-Bremek. (Ergül \& Dülger 2000d; Oran et al. 2006)

\section{Echinosteliaceae}

Echinostelium apitectum K.D. Whitney (Ergül et al. 2005b), E. arboreum H.W. Keller \& T.E. Brooks (Ergül \& Dülger 2000d; Ergül et al. 2005b), E. coelocephalum T.E. Brooks \& H.W. Keller (Ergül \& Dülger 2000d), $E$. colliculosum K.D. Whitney \& H.W. Keller (Härkönen \& Uotila 1983; Ergül 
\& Dülger 2000d), E. corynophorum K.D. Whitney (Ergül \& Dülger 2000d), E. cribrarioides Alexop. (Ergül \& Dülger 2000d), E. elachiston Alexop. (Härkönen 1988; Ergül \& Dülger 2000d), E. fragile Nann.-Bremek. (Ergül \& Dülger 2000d), E. ladoi Pando (Oran \& Ergül 2004), E. minutum de Bary (Härkönen \& Uotila 1983; Härkönen 1988; Ergül \& Dülger 1998; Ergül \& Dülger 2000d; Ocak \& Hasenekoğlu 2003a, 2005; Ergül et al. 2005b; Oran et al. 2006; Yağız \& Afyon 2006a; Baba \& Tamer 2008a; Ergül \& Akgül 2011; Baba et al. 2013b; Baba \& Zümre 2015; Ocak 2015; Oskay \& Tüzün 2015)

\section{Liceida}

\section{Cribrariaceae}

Cribraria argillacea (Pers. ex J.F. Gmel.) Pers. (Ergül \& Dülger 2002c; Ergül et al. 2005a; Ocak \& Hasenekoğlu 2005; Demirel et al. 2006; Baba \& Tamer 2008a; Ergül \& Akgül 2011; Baba et al. 2013b, 2015), C. aurantiaca Schrad. (Ergül \& Dülger 2000d; Ergül et al. 2005a; Oran et al. 2006; Yağız \& Afyon 2006a), C. cancellata (Batsch) Nann.-Bremek. (Ergül \& Gücin 1993; Ergül \& Dülger 1998; Ergül \& Dülger 2000d; Yağız et al. 2002; Ocak \& Hasenekoğlu 2003a, 2005; Yağız \& Afyon 2003, 2006a; Ergül et al. 2005a, b; Demirel et al. 2006; Oran et al. 2006; Baba \& Tamer 2008a; Ergül \& Akgül 2011; Baba et al. 2013b, 2015; Eroğlu \& Kaşik 2013a; Baba \& Zümre 2015; Oskay \& Tüzün 2015), C. confusa Nann.-Bremek. \& Y. Yamam. (Ergül et al. 2005b), C. elegans Berk. \& M.A. Curtis (Ergül \& Dülger 2002c; Ergül et al. 2005b; Baba et al. 2013b), C. intricata Schrad. (Ocak \& Hasenekoğlu 2005; Demirel et al. 2006; Yağız \& Afyon 2006a; Baba \& Tamer 2008a; Baba et al. 2013b, 2015), C. languescens Rex (Yağız \& Afyon 2006a, c, 2007a; Baba \& Tamer 2008a), C. macrocarpa Schrad. (Ocak \& Hasenekoğlu 2005; Baba et al. 2013b), C. microcarpa (Schrad.) Pers. (Ergül \& Dülger 2002c; Ergül et al. 2005b; Ocak \& Hasenekoğlu 2005; Oran et al. 2006; Baba et al. 2013b), C. minutissima Schwein. (Ergül \& Dülger 2000d; Baba et al. 2013b), C. mirabilis (Rostaf.) Massee (Yağız \& Afyon 2007b; Demirel et al. 2010; Ocak 2015), C. oregana H.C. Gilbert (Eroğlu \& Kaşik 2013b), C. persoonii Nann.Bremek. (Yağı \& Afyon 2003, 2005, 2006a), C. piriformis Schrad. (Yağ Afyon 2007b; Baba \& Tamer 2008a; Demirel et al. 2010; Baba et al. 2013b), C. tenella Schrad. (Ergül \& Dülger 2002c; Ergül et al. 2005a), C. violacea Rex (Härkönen \& Uotila 1983; Ergül \& Dülger 1998; Ergül \& Dülger 2000d; Ergül et al. 2005b; Oran et al. 2006; Yağız \& Afyon 2006a; Baba et al. 2013b; Eroğlu \& Kaşik 2013a), C. vulgaris Schrad. (Oran \& Ergül 2004; Demirel et al. 2006; Yağız \& Afyon 2006a; Baba \& Tamer 2008a; Baba 2012; Baba et al. 2013b, 2015; Oskay \& Tüzün 2015)

Lindbladia tubulina Fr. (Ergül \& Dülger 2002c; Ergül et al. 2005b)

\section{Dictydiaethaliaceae}

Dictydiaethalium plumbeum (Schumach.) Rostaf ex Lister (Baba et al. 2008, 2013b; Baba 2012) 


\section{Liceaceae}

Licea belmontiana Nann.-Bremek. (Oran \& Ergül 2004; Ergül et al. 2005b; Yağız \& Afyon 2006a), L. biforis Morgan (Ocak \& Hasenekoğlu 2005; Yağız \& Afyon 2006a; Baba 2012; Baba et al. 2013b; Baba \& Zümre 2015), L. castanea G. Lister (Ergül \& Dülger 2000d; Ocak \& Hasenekoğlu 2003a; Ergül et al. 2005b; Oran et al. 2006; Yağız \& Afyon 2006a; Baba \& Tamer 2008a; Ergül \& Akgül 2011; Baba et al. 2013b; Baba \& Zümre 2015; Ocak 2015; Oskay \& Tüzün 2015), L. denudescens H.W. Keller \& T.E. Brooks (Härkönen \& Uotila 1983; Ergül \& Dülger 2000d; Ocak \& Hasenekoğlu 2003a), L. inconspicua T.E. Brooks \& H.W. Keller (Oran \& Ergül 2004; Ergül et al. 2005b), L. kleistobolus G.W. Martin (Härkönen \& Uotila 1983; Härkönen 1988; Ergül \& Dülger 2000d; Ergül et al. 2005b; Ocak \& Hasenekoğlu 2005; Oran et al. 2006; Baba \& Tamer 2008a; Baba et al. 2013b; Oskay \& Tüzün 2015), L. marginata Nann.-Bremek. (Yağız \& Afyon 2007b; Demirel et al. 2010), L. minima Fr. (Ergül \& Dülger 2000d; Ocak \& Hasenekoğlu 2005; Demirel et al. 2006; Oran et al. 2006; Yağız \& Afyon 2006a; Baba \& Tamer 2008a; Baba et al. 2013b; Ocak 2015; Oskay \& Tüzün 2015), L. operculata (Wingate) G.W. Martin (Ergül \& Dülger 2000d; Ocak \& Hasenekoğlu 2005; Oran et al. 2006; Yağız \& Afyon 2006a), L. parasitica (Zukal) G.W. Martin (Ergül \& Dülger 2000d; Ergül et al. 2005b; Ocak \& Hasenekoğlu 2005; Oran et al. 2006; Eroğlu \& Kaşik 2013a), L. pedicellata (H.C. Gilbert) H.C. Gilbert (Ergül \& Dülger 2000d; Oran et al. 2006; Baba \& Tamer 2008a; Baba et al. 2013b), L. perexigua T.E. Brooks \& H.W. Keller (Oran \& Ergül 2004), L. punctiformis G.W. Martin (Ergül \& Dülger 2000d; Ergül et al. 2005b; Oran et al. 2006), L. pusilla Schrad. (Ergül \& Dülger 2000d; Ergül et al. 2005b; Ocak \& Hasenekoğlu 2005; Oran et al. 2006; Demirel et al. 2006; Baba 2012; Eroğlu \& Kaşik 2013a), L. pygmaea (Meyl.) Ing (Ergül \& Dülger 1998, 2000d), L. scyphoides T.E. Brooks \& H.W. Keller (Ergül et al. 2005b), L. synsporos Nann.-Bremek. (Ocak \& Hasenekoğlu 2003b), L. tenera E. Jahn (Ergül \& Dülger 2000d; Ocak \& Hasenekoğlu 2003a; Ergül et al. 2005b; Baba et al. 2013b), L. testudinacea Nann.-Bremek. (Eroğlu et al. 2015), L. tuberculata G. W. Martin (Ocak \& Hasenekoğlu 2003a), L. variabilis Schrad. (Ocak \& Hasenekoğlu 2003b; Baba \& Tamer 2008a; Baba 2012)

\section{Tubiferaceae}

Lycogala epidendrum (L.) Fr. (Lohwag 1957, 1964; Selik 1964; Härkönen 1988; Gücin et al. 1996b; Ergül \& Dülger 1998, 2000d; Kaya \& Demirel 1998; Pekşen \& Karaca 2000, 2003; Türkekul 2003; Demirel, K. et al. 2004; Ergül et al. 2005a, b; Ocak \& Hasenekoğlu 2005; Demirel, G. et al. 2006; Baba \& Tamer 2008a; Ergül \& Akgül 2011; Baba et al. 2013b, 2015; Baba \& Zümre 2015; Ocak 2015; Oskay \& Tüzün 2015), L. exiguum Morgan (Oran \& Ergül 2004; Ocak \& Hasenekoğlu 2005; Ergül \& Akgül 2011; Eroğlu \& Kaşik 2013a), L. flavofuscum (Ehrenb.) Rostaf. (Ergül \& Dülger 2000d; Ergül et al. 2005a, b) 
Reticularia jurana Meyl. (Ergül et al. 2005b; Eroğlu \& Kaşik 2013a), R. liceoides (Lister) Nann.-Bremek. (Ergül \& Oran 2005), R. lycoperdon Bull. (Demirel \& Uzun 2002; Kaya \& Demirel 1998; Demirel \& Uzun 2002; Baba et al. 2013b, 2015; Eroğlu \& Kaşik 2013a; Ocak 2015), R. splendens Morgan (Ergül 1995; Gücin et al. 1996b; Ergül \& Dülger 2000d; Gücin \& Dülger 2007; Ergül \& Akgül 2011)

Tubifera ferruginosa (Batsch) J.F. Gmel. (Ergül \& Dülger 2002c; Ergül et al. 2005b; Ocak \& Hasenekoğlu 2005; Yağız \& Afyon 2006a; Ergül \& Akgül 2011)

\section{Physarida}

\section{Didymiaceae}

Diderma carneum Nann.-Bremek. (Baba \& Tamer 2008b; Baba et al. 2013b, 2015), D. chondrioderma (de Bary \& Rostaf.) G. Lister (Ergül 1992; Ergül \& Dülger 1998, 2000d; Ergül et al. 2005b), D. cinereum Morgan (Oran \& Ergül 2015), D. crustaceum Peck (Dülger 2008b), D. deplanatum Fr. (Baba 2012), D. hemisphaericum (Bull.) Hornem. (Ergül \& Dülger 2000d; Ocak \& Hasenekoğlu 2005; Baba et al. 2013b, 2015; Ocak 2015), D. niveum (Rostaf.) E. Sheld. (Ergül et al. 2005b), D. radiatum (L.) Morgan (Baba et al. 2013b), D. testaceum (Schrad.) Pers. (Ergül \& Dülger 2002c; Ergül et al. 2005b; Ocak 2015), D. umbilicatum Pers. (Oran \& Ergül 2015)

Didymium anellus Morgan (Ocak \& Hasenekoğlu 2005; Baba et al. 2013b), D. annulisporum H.W. Keller \& Schokn. (Eroğlu \& Kaşik 2013a), D. bahiense Gottsb. (Ergül \& Dülger 2002c; Ergül et al. 2005b; Baba \& Tamer 2008a; Baba et al. 2013b, 2015), D. crustaceum Fr. (Ocak \& Hasenekoğlu 2003a), D. difforme (Pers.) Gray (Ergül \& Dülger 2000d; Yağız \& Afyon 2006a; Baba \& Tamer 2008a; Baba 2012; Baba et al. 2013b, 2015; Eroğlu \& Kaşik 2013a; Baba \& Zümre 2015; Oskay \& Tüzün 2015), D. dubium Rostaf. (Baba et al. 2013b), D. eximium Peck (Ergül \& Dülger 2002c; Ergül et al. 2005b), D. floccosum G.W. Martin, K.S. Thind \& Rehill (Ergül \& Dülger 1998, 2000d; Dülger et al. 2006), D. iridis (Ditmar) Fr. (Ocak \& Hasenekoğlu 2003b; Baba \& Tamer 2008a), D. karstensii Nann.-Bremek. (Demirel \& Kaşık 2012; Eroğlu \& Kaşik 2013a), D. lenticulare K.S. Thind \& T.N. Lakh. (Oran \& Ergül 2015), D. megalosporum Berk \& M.A. Curtis (Baba 2012; Baba et al. 2013b, 2015), D. melanospermum (Pers.) T. Macbr. (Baba et al. 2008, 2013b, 2015; Baba 2012), D. minus (Lister) Morgan (Ergül \& Dülger 2000d), D. nigripes (Link) Fr. (Ergül \& Dülger 2002c; Ergül et al. 2005b; Ocak \& Hasenekoğlu 2005), D. quitense (Pat.) Torrend (Härkönen \& Uotila 1983; Ergül \& Dülger 2000d; Ocak \& Hasenekoğlu 2003a, 2005), D. squamulosum (Alb. \& Schwein.) Fr. (Härkönen \& Uotila 1983; Ergül \& Dülger 2000d; Ocak \& Hasenekoğlu 2003a, 2005; Baba \& Tamer 2008a; Baba 2012; Baba et al. 2013b, 2015; Eroğlu \& Kaşik 2013a; Baba \& Zümre 2015; Oskay \& Tüzün 2015), D. sturgisii Hagelst. (Ergül et al. 2005b), D. trachysporum 
G. Lister (Demirel \& Kaşık 2012; Baba et al. 2013b, 2014; Eroğlu \& Kaşik 2013a), D. vaccinum (Durieu \& Mont.) Buchet (Eroğlu et al. 2015)

Mucilago crustacea F.H. Wigg. (Ergül \& Dülger 2000d, 2002a)

\section{Physaraceae}

Badhamia affinis Rostaf. (Härkönen \& Uotila 1983; Ergül et al. 2005b; Yağız \& Afyon 2005), B. capsulifera (Bull.) Berk. (Ergül \& Dülger 2002c; Yağız \& Afyon 2005, 2006a), B. dubia Nann.-Bremek. (Dülger 2008c; Baba et al. 2013b, 2015), B. foliicola Lister (Härkönen \& Uotila 1983; Ergül \& Dülger 1998, 2000d; Ocak \& Hasenekoğlu 2003a, 2005; Ergül et al. 2005b; Yağız \& Afyon 2005; Oran et al. 2006; Baba \& Tamer 2008a; Ergül \& Akgül 2011; Baba et al. 2013b; Eroğlu \& Kaşik 2013a), B. goniospora Meyl. (Ergül et al. 2005b; Yağız \& Afyon 2006a), B. iowensis T. Macbr. (Eroğlu \& Kaşik 2013b), B. macrocarpa (Ces.) Rostaf. (Gücin \& Öner 1986; Ergül \& Dülger 2000d; Ocak \& Hasenekoğlu 2003a; Ergül et al. 2005b; Demirel et al. 2006; Oran et al. 2006; Yağız \& Afyon 2006a; Baba \& Tamer 2008a; Baba et al. 2013b, 2015; Eroğlu \& Kaşik 2013a), B. melanospora Speg. (Dülger 2008b; Eroğlu \& Kaşik 2013a), B. nitens Berk. (Ergül \& Dülger 2000d; Ergül et al. 2005b; Oran et al. 2006; Zümre et al. 2013), B. panicea (Fr.) Rostaf. (Härkönen \& Uotila 1983; Ergül \& Dülger 2000d; Ocak \& Hasenekoğlu 2003a, 2005; Demirel et al. 2006; Yağız \& Afyon 2006a; Baba et al. 2013b; Eroğlu \& Kaşik 2013a), B. papaveracea Berk. \& Ravenel (Ergül et al. 2005b), B. populina Lister \& G. Lister (Ergül et al. 2005b; Baba \& Tamer 2008a), B. utricularis (Bull.) Berk. (Oran \& Ergül 2004; Dülger \& Gönüz 2005; Yağız \& Afyon 2006a; Baba \& Tamer 2008a; Ergül \& Akgül 2011; Baba et al. 2013b), B. versicolor Lister (Härkönen \& Uotila 1983; Ergül \& Dülger 2000d; Ergül et al. 2005b; Oran et al. 2006), B. viridescens Meyl. (Ergül 1992; Ergül \& Dülger 2000d)

Badhamiopsis ainoae (Yamash.) T.E. Brooks \& H.W. Keller (Ergül \& Dülger 2000d; Ocak \& Hasenekoğlu 2003a; Ergül et al. 2005b; Eroğlu \& Kaşik 2013a)

Craterium concinnum Rex (Ocak \& Hasenekoğlu 2005; Eroğlu \& Kaşik 2013a), C. leucocephalum (Pers. ex J.F. Gmel.) Ditmar (Ergül \& Oran 2005; Eroğlu \& Kaşik 2013a), C. minutum (Leers) Fr. (Ergül \& Dülger 2002c; Ergül et al. 2005a)

Fuligo cinerea (Schwein.) Morgan (Ocak 2015), F. septica (L.) F.H. Wigg. (Ergül \& Gücin 1994; Ergül \& Dülger 2000d, 2002c; Ergül et al. 2005a, b; Ocak \& Hasenekoğlu 2005; Oran et al. 2006; Yağız \& Afyon 2007b; Baba \& Tamer 2008a; Ergül \& Akgül 2011; Eroğlu \& Kaşik 2013a; Ocak 2015)

Leocarpus fragilis (Dicks.) Rostaf. (Ergül \& Dülger 1998, 2000d; Ocak \& Hasenekoğlu 2005; Yağız \& Afyon 2005; Ergül \& Akgül 2011; Ocak 2015)

Physarum albescens Ellis ex T. Macbr. (Baba \& Gelen 2014), Ph. album (Bull.) Chevall. (Ergül \& Dülger 1998, 2000d; Yağız et al. 2002; Ergül et al. 2005a, b; Ocak \& Hasenekoğlu 2005; Demirel et al. 2006; Dülger et al. 2006; Oran 
et al. 2006; Yağız \& Afyon 2006a; Baba \& Tamer 2008a; Ergül \& Akgül 2011; Baba et al. 2013b, 2015; Baba 2015; Baba \& Zümre 2015; Ocak 2015; Oskay \& Tüzün 2015), Ph. auriscalpium Cooke (Härkönen 1988; Ergül et al. 2005b; Oran et al. 2006; Baba \& Tamer 2008a; Baba et al. 2013b; Eroğlu \& Kaşik 2013a; Baba 2015), Ph. bitectum G. Lister (Härkönen \& Uotila 1983; Ergül \& Dülger 2000d; Baba 2015), Ph. cinereum (Batsch) Pers. (Härkönen 1988; Ergül \& Dülger 2000d; Ocak \& Hasenekoğlu 2003a, 2005; Baba \& Tamer 2008a; Baba et al. 2013b, 2015; Eroğlu \& Kaşik 2013a; Baba 2015; Baba \& Zümre 2015; Oskay \& Tüzün 2015), Ph. compressum Alb. \& Schwein. (Ergül \& Dülger 2000d; Baba \& Tamer 2008a; Baba 2015; Baba et al. 2013b, 2015), Ph. confertum T. Macbr. (Baba 2008, 2015; Baba et al. 2013b; Eroğlu \& Kaşik 2013a; Baba \& Zümre 2015), Ph. contextum (Pers.) Pers. (Härkönen \& Uotila 1983; Ergül \& Dülger 2000d; Baba et al. 2013b, 2015; Baba 2015), Ph. decipiens M.A. Curtis (Härkönen \& Uotila 1983; Ergül \& Dülger 2000d; Yağı et al. 2002; Ocak \& Hasenekoğlu 2003a; Ergül et al. 2005b; Yağız \& Afyon 2005; Dülger et al. 2006; Baba 2015), Ph. didermoides (Pers.) Rostaf. (Demirel \& Kaşık 2012; Eroğlu \& Kaşik 2013a; Baba 2015), Ph. famintzinii Rostaf. (Eroğlu et al. 2014, 2015; Baba 2015), Ph. flavicomum Berk. (Ergül \& Dülger 2002c; Ergül et al. 2005a; Oran et al. 2006; Ergül \& Akgül 2011; Baba et al. 2013b; Baba 2015), Ph. galbeum Wingate (Dülger 2008a; Baba 2015), Ph. globuliferum (Bull.) Pers. (Ergül et al. 2005b; Baba 2015), Ph. gyrosum Rostaf. (Demirel \& Kaşık 2012; Eroğlu \& Kaşik 2013a; Baba 2015), Ph. javanicum Racib. (Baba 2015), Ph. leucophaeum Fr. (Ergül \& Dülger 2000d; Ocak \& Hasenekoğlu 2003a; Yağız \& Afyon 2006a; Baba \& Tamer 2008a; Ergül \& Akgül 2011; Baba et al. 2013b; Eroğlu \& Kaşik 2013a; Baba 2015), Ph. leucopus Link (Ocak \& Hasenekoğlu 2003a; Baba 2015), Ph. licheniforme (Szabó ex Schwein.) Lado (Baba et al. 2012; Baba 2015), Ph. luteolum Peck (Oran \& Ergül 2004; Baba 2015), Ph. notabile T. Macbr. (Ergül \& Dülger 2002c; Ocak \& Hasenekoğlu 2003a; Ergül et al. 2005b; Baba \& Tamer 2008a; Baba et al. 2013b; Eroğlu \& Kaşik 2013a; Baba 2015; Ocak 2015), Ph. nudum T. Macbr. (Eroğlu \& Kaşik 2013b; Ocak 2015), Ph. oblatum T. Macbr. (Ergül \& Dülger 2000d; Baba \& Tamer 2008a; Baba et al. 2013b; Eroğlu \& Kaşik 2013a; Baba 2015), Ph. ovisporum G. Lister (Ergül \& Dülger 2002c; Baba et al. 2013b; Baba 2015), Ph. perfectum M. Peck (Dülger \& Duman 2010; Baba 2015), Ph. pulcherrimum Berk. \& Ravenel (Ergül \& Oran 2005; Baba 2015), Ph. pusillum (Berk. \& M.A. Curtis) G. Lister (Ergül \& Dülger 1998, 2000d; Yağız et al. 2002; Ocak \& Hasenekoğlu 2003a; Dülger et al. 2006; Oran et al. 2006; Baba 2015), Ph. robustum (Lister) Nann.-Bremek. (Yağız \& Afyon 2007b; Demirel et al. 2010; Baba 2015), Ph. serpula Morgan (Oran \& Ergül 2015), Ph. tropicale T. Macbr. (Baba \& Gelen 2014), Ph. vernum Sommerf. (Oran \& Ergül 2004; Baba 2015), Ph. viride (Bull.) Pers. (Ergül \& Dülger 1998, 2000d; Ergül et al. 2005a; Ocak \& Hasenekoğlu 2005; Oran et 
al. 2006; Yağız \& Afyon 2006a; Baba \& Tamer 2008a; Ergül \& Akgül 2011; Baba et al. 2013b, 2015; Eroğlu \& Kaşik 2013a; Baba 2015)

Protophysarum phloiogenum M. Blackw. \& Alexop. (Oran \& Ergül 2004)

Willkommlangea reticulata (Alb.\& Schwein.) Kuntze (Baba \& Tamer 2008b)

\section{Stemonitida}

Stemonitidaceae

Amaurochaete atra (Alb. \& Schwein.) Rostaf. (Sümer 1982; Abatay 1989; Ergül \& Dülger 2000d; Yağız \& Afyon 2007b; Baba \& Tamer 2008a), A. comata G. Lister \& Brândză (Eroğlu \& Kaşik 2013b), A. tubulina (Alb. \& Schwein.) T. Macbr. (Oran \& Ergül 2015)

Collaria arcyrionema (Rostaf.) Nann.-Bremek. ex Lado (Oran \& Ergül 2004; Ergül et al. 2005b), C. lurida (Lister) Nann.-Bremek. (Ergül \& Dülger 2000d; Ocak \& Hasenekoğlu 2003a, 2005; Demirel et al. 2006; Baba \& Tamer 2008a; Baba 2012; Baba et al. 2013b, 2015; Oskay \& Tüzün 2015), C. rubens (Lister) Nann.-Bremek. (Härkönen 1988; Ocak 2015)

Comatricha alta Preuss (Eroğlu \& Kaşik 2013b), C. elegans (Racib.) G. Lister (Härkönen \& Uotila 1983; Ergül \& Dülger 2000d; Ocak \& Hasenekoğlu 2005; Baba \& Tamer 2008a; Baba 2012; Baba et al. 2013b; Eroğlu \& Kaşik 2013a), C. ellae Härk. (Ergül \& Dülger 2000d; Yağız \& Afyon 2005; Oran et al. 2006; Baba \& Tamer 2008a; Baba 2012, Baba et al. 2013b, 2015; Eroğlu \& Kaşik 2013a; Baba \& Zümre 2015; Ocak 2015; Oskay \& Tüzün 2015), $C$. laxa Rostaf. (Härkönen \& Uotila 1983; Ergül \& Dülger 1998, 2000d; Ergül et al. 2005b; Demirel et al. 2006; Dülger et al. 2006; Oran et al. 2006; Baba \& Tamer 2008a; Baba et al. 2013b, 2015; Eroğlu \& Kaşik 2013a; Baba \& Zümre 2015), C. longipila Nann.-Bremek. (Oran \& Ergül 2015), C. nigra (Pers. ex J.F. Gmel.) J. Schröt. (Härkönen \& Uotila 1983; Ergül \& Dülger 1998, 2000d; Yağız et al. 2002; Ocak \& Hasenekoğlu 2003a, 2005; Ergül et al. 2005b; Demirel et al. 2006; Dülger et al. 2006; Oran et al. 2006; Yağız \& Afyon 2006a; Baba \& Tamer 2008a; Baba et al. 2013b, 2015; Eroğlu \& Kaşik 2013a; Eroğlu et al. 2014; Baba \& Zümre 2015; Ocak 2015; Oskay \& Tüzün 2015), C. pulchella (C. Bab.) Rostaf. (Ergül \& Dülger 2002b; Yağız et al. 2002; Ocak \& Hasenekoğlu 2005; Demirel et al. 2006; Yağız \& Afyon 2006a; Baba \& Tamer 2008a; Baba et al. 2013b; Eroğlu \& Kaşik 2013a; Eroğlu et al. 2014; Baba \& Zümre 2015), C. pulchelloides Nann.-Bremek. (Eroğlu \& Kaşik 2013a), C. rigidireta Nann.-Bremek. (Eroğlu \& Kaşik 2013a), C. suksdorfii Ellis \& Everh. (Dülger et al. 2007), C. tenerrima (M.A. Curtis) G. Lister (Ocak \& Hasenekoğlu 2005; Oran et al. 2006; Baba 2012; Baba et al. 2013b)

Diachea leucopodia (Bull.) Rostaf. (Härkönen \& Uotila 1983; Ergül \& Dülger 2000d; Oran et al. 2006; Baba et al. 2015)

Diacheopsis minuta Nann.-Bremek. \& Y. Yamam. (Ocak 2015)

Enerthenema papillatum (Pers.) Rostaf. (Härkönen \& Uotila 1983; Ergül \& Dülger 2000d; Ocak \& Hasenekoğlu 2003a, 2005; Yağız \& Afyon 2005, 
2006a; Demirel et al. 2006; Oran et al. 2006; Baba \& Tamer 2008a; Ergül \& Akgül 2011; Baba et al. 2013b, 2015; Eroğlu \& Kaşik 2013a; Eroğlu et al. 2014; Baba \& Zümre 2015; Oskay \& Tüzün 2015)

Lamproderma arcyrioides (Sommerf.) Rostaf. (Härkönen 1988; Ergül \& Dülger 2000d; Ergül \& Akgül 2011; Baba et al. 2013b), L. columbinum (Pers.) Rostaf. (Oran \& Ergül 2004), L. laxum H. Neubert (Baba et al. 2013b), L. scintillans (Berk. \& Broome) Morgan (Ocak \& Hasenekoğlu 2005; Baba et al. 2013b, 2015)

Macbrideola cornea (G. Lister \& Cran) Alexop. (Härkönen \& Uotila 1983; Härkönen 1988; Ergül \& Dülger 2000d; Ergül et al. 2005b; Ocak \& Hasenekoğlu 2005; Yağız \& Afyon 2005, 2006a; Oran et al. 2006; Baba \& Tamer 2008a; Baba et al. 2013b; Oskay \& Tüzün 2015), M. decapillata H.C. Gilbert (Ergül \& Dülger 2000d; Yağız et al. 2002; Ergül et al. 2005b; Dülger et al. 2006; Yağız \& Afyon 2006a; Baba \& Tamer 2008a; Baba et al. 2013b; Baba \& Zümre 2015), M. dubia Nann.-Bremek. \& Y. Yamam. (Ergül et al. 2005b; Ocak 2015), M. macrospora (Nann.-Bremek.) Ing (Yağı \& Afyon 2006a, c, 2007a; Baba et al. 2013b), M. martinii (Alexop. \& Beneke) Alexop. (Oran \& Ergül 2004; Baba \& Tamer 2008a), M. scintillans H.C. Gilbert (Ergül \& Dülger 2002c; Ergül et al. 2005b), M. synsporos (Alexop.) Alexop. (Ergül \& Dülger 2000d; Ergül et al. 2005b; Baba \& Tamer 2008a; Ergül \& Akgül 2011; Ocak 2015)

Meriderma cribrarioides (Fr.) Mar. Mey. \& Poulain (Baba 2012)

Paradiacheopsis acanthodes (Alexop.) Nann.-Bremek. (Ergül \& Dülger 2000a; Ocak \& Hasenekoğlu 2005; Oran et al. 2006), P. cribrata Nann.-Bremek. (Oran \& Ergül 2004), P. fimbriata (G. Lister \& Cran) Hertel ex Nann.Bremek. (Härkönen \& Uotila 1983; Härkönen 1988; Ergül \& Dülger 2000d; Oran et al. 2006; Baba \& Tamer 2008a), P. microcarpa (Meyl.) D.W. Mitch. ex Ing (Oran \& Ergül 2004), P. rigida (Brândză) Nann.-Bremek. (Ergül \& Dülger 2000a; Oran \& Ergül 2004; Ergül \& Akgül 2011; Baba et al. 2013b), P. solitaria (Nann.-Bremek.) Nann.-Bremek. (Ergül \& Dülger 2000a; Oran et al. 2006; Ergül \& Akgül 2011)

Stemonaria irregularis (Rex) Nann.-Bremek., R. Sharma \& Y. Yamam. (Ergül \& Dülger 2002c; Yağız \& Afyon 2007b)

Stemonitis axifera (Bull.) T. Macbr. (Ergül \& Dülger 2002c; Ergül et al. 2005a, b; Ocak \& Hasenekoğlu 2005; Oran et al. 2006; Baba \& Tamer 2008a; Baba et al. 2013b, 2015; Ocak 2015), S. flavogenita E. Jahn (Härkönen \& Uotila 1983; Ergül \& Dülger 2000d; Ergül et al. 2005a, b; Yağız \& Afyon 2005; Oran et al. 2006; Baba \& Tamer 2008a; Baba et al. 2013b, 2015; Ocak 2015), S. foliicola Ing (Baba 2008, 2012; Baba et al. 2013b; Eroğlu \& Kaşik 2013a), S. fusca Roth (Härkönen \& Uotila 1983; Ergül \& Dülger 1998, 2000d; Ergül et al. 2005a; Ocak \& Hasenekoğlu 2005; Yağız \& Afyon 2005; Dülger et al. 2006; Oran et al. 2006; Baba \& Tamer 2008a; Baba et al. 2013b, 2015; Eroğlu \& Kaşik 2013a; Eroğlu et al. 2014; Baba \& Zümre 2015; Ocak 2015; 
Oskay \& Tüzün 2015), S. herbatica Peck (Ergül \& Dülger 2002c; Yağız et al. 2002; Ergül et al. 2005a, b; Ocak \& Hasenekoğlu 2005; Oran et al. 2006; Baba \& Tamer 2008a; Baba et al. 2013b, 2015; Eroğlu \& Kaşik 2013a), S. inconspicua Nann.-Bremek. (Oran \& Ergül 2015), S. mediterraneensis H.H. Doğan \& Eroğlu (Doğan \& Eroğlu 2014), S. pallida Wingate (Ergül \& Dülger 2002c; Ergül et al. 2005a, b; Yağız \& Afyon 2006a; Baba \& Tamer 2008a; Eroğlu \& Kaşik 2013a), S. smithii T. Macbr. (Ergül \& Dülger 2002c; Ergül et al. 2005a, b; Ocak \& Hasenekoğlu 2005; Demirel et al. 2006; Oran et al. 2006; Baba \& Tamer 2008a), S. splendens Rostaf. (Gücin \& Öner 1986; Ergül \& Dülger 2000d; Ergül et al. 2005a, b; Yağız \& Afyon 2005; Baba et al. 2013b), S. virginiensis Rex (Ergül \& Dülger 2002c; Ergül et al. 2005a, b; Yağız \& Afyon 2006a; Ocak 2015)

Stemonitopsis amoena (Nann.-Bremek.) Nann.-Bremek. (Yağı \& Afyon 2005, 2006b; Baba \& Tamer 2008a; Baba 2012; Baba et al. 2013b, 2015; Eroğlu \& Kaşik 2013a; Baba \& Zümre 2015; Oskay \& Tüzün 2015), S. gracilis (G. Lister) Nann.-Bremek. (Yağız \& Afyon 2005, 2006b; Eroğlu \& Kaşik 2013a), S. hyperopta (Meyl.) Nann.-Bremek. (Ergül \& Dülger 2000d; Ocak \& Hasenekoğlu 2003a, 2005; Yağız \& Afyon 2006a; Baba \& Tamer 2008a; Baba et al. 2013b, 2015), S. microspora (Lister) Nann.-Bremek. (Ergül \& Dülger 2000c; Yağız \& Afyon 2006a), S. reticulata (H.C. Gilbert) Nann.-Bremek. \& Y. Yamam. (Baba \& Tamer 2008a; Baba et al. 2013b, 2015), S. subcaespitosa (Peck) Nann.-Bremek. (Ergül \& Dülger 2002c; Oran et al. 2006; Baba et al. 2013b, 2015), S. typhina (F.H. Wigg.) Nann.-Bremek. (Härkönen 1988; Ergül \& Dülger 2000c, d; Ergül et al. 2005a, b; Oran et al. 2006; Ergül \& Akgül 2011; Baba et al. 2013b)

Symphytocarpus confluens (Cooke \& Ellis) Ing \& Nann.-Bremek. (Eroğlu \& Kaşik 2013b; Oran \& Ergül 2015), S. flaccidus (Lister) Ing \& Nann.-Bremek. (Ergül \& Dülger 1999, 2000d), S. impexus Ing \& Nann.-Bremek. (Baba et al. 2008), S. trechispora (Berk. ex Torrend) Nann.-Bremek (Baba et al. 2013b)

\section{Trichiida}

Arcyriaceae

Arcyria affinis Rostaf. (Ergül \& Dülger 2002c; Ergül et al. 2005a; Yağız \& Afyon 2006a; Baba et al. 2013b, 2015), A. annulifera G. Lister \& Torrend (Oran \& Ergül 2004; Ocak 2015; Oskay \& Tüzün 2015), A. cinerea (Bull.) Pers. (Härkönen \& Uotila 1983; Härkönen 1988; Ergül \& Dülger 2000d; Ocak \& Hasenekoğlu 2003a, 2005; Ergül et al. 2005a, b; Demirel et al. 2006; Dülger et al. 2006; Oran et al. 2006; Yağız \& Afyon 2006a; Baba \& Tamer 2008a; Ergül \& Akgül 2011; Baba et al. 2013b, 2015; Eroğlu \& Kaşik 2013a; Eroğlu et al. 2014; Baba \& Zümre 2015; Ocak 2015; Oskay \& Tüzün 2015), A. denudata (L.) Wettst. (Härkönen 1988; Ergül \& Dülger 2000d; Ergül et al. 2005a, b; Ocak \& Hasenekoğlu 2005; Oran et al. 2006; Baba \& Tamer 2008a; Ergül \& Akgül 2011; Baba et al. 2013b, 2015; Eroğlu \& Kaşik 2013a; Baba \& Zümre 
2015), A. ferruginea Saut. (Ergül \& Dülger 2002c; Oran et al. 2006; Baba et al. 2013b; Ocak 2015; Oskay \& Tüzün 2015), A. globosa Schwein. (Ocak \& Hasenekoğlu 2005; Yağız \& Afyon 2005, 2006a; Demirel et al. 2006; Baba \& Tamer 2008a; Eroğlu \& Kaşik 2013a; Oskay \& Tüzün 2015), A. imperialis (G. Lister) Q. Wang \& Yu Li (Oran \& Ergül 2004; Yağız \& Afyon 2007b), $A$. incarnata (Pers. ex J.F. Gmel.) Pers. (Ergül \& Dülger 2000d; Yağı et al. 2002; Ergül et al. 2005a, b; Ocak \& Hasenekoğlu 2005; Yağız \& Afyon 2005, 2006a, c; Demirel et al. 2006; Dülger et al. 2006; Oran et al. 2006; Baba \& Tamer 2008a; Ergül \& Akgül 2011; Baba et al. 2013b, 2015; Eroğlu \& Kaşik 2013a; Eroğlu et al. 2014; Baba \& Zümre 2015), A. insignis Kalchbr. \& Cooke (Ergül \& Dülger 2000d; Ergül et al. 2005a, b; Ocak \& Hasenekoğlu 2005; Baba \& Tamer 2008a; Ergül \& Akgül 2011; Oskay \& Tüzün 2015), A. magna Rex (Ocak \& Hasenekoğlu 2005; Demirel et al. 2006), $A$. major (G. Lister) Ing (Yağız \& Afyon 2005), A. minuta Buchet (Ergül \& Dülger 2000b, d, 2002c; Oran et al. 2006; Baba 2012; Baba et al. 2013b, 2015; Ocak 2015), A. nigella Emoto (Ocak \& Hasenekoğlu 2005; Oskay \& Tüzün 2015), A. obvelata (Oeder) Onsberg (Ergül \& Dülger 1998, 2000d; Ocak \& Hasenekoğlu 2003a, 2005; Ergül et al. 2005a, b; Yağız \& Afyon 2005, 2006a, 2007b; Demirel et al. 2006; Dülger et al. 2006, Oran et al. 2006; Baba \& Tamer 2008a; Ergül \& Akgül 2011; Baba et al. 2013b, 2015; Eroğlu \& Kaşik 2013a; Baba \& Zümre 2015; Ocak 2015; Oskay \& Tüzün 2015), A. occidentalis (T. Macbr.) G. Lister (Dülger et al. 2005), A. oerstedii Rostaf. (Dülger et al. 2006; Yağız \& Afyon 2006a, c), A. pomiformis (Leers) Rostaf. (Härkönen \& Uotila 1983; Härkönen 1988; Ergül \& Dülger 2000d; Ocak \& Hasenekoğlu 2003a, 2005; Ergül et al. 2005a; Demirel et al. 2006; Yağı \& Afyon 2006a; Baba \& Tamer 2008a; Baba et al. 2013b, 2015; Eroğlu \& Kaşik 2013a; Baba \& Zümre 2015; Ocak 2015; Oskay \& Tüzün 2015), A. stipata (Schwein.) Lister (Ergül \& Dülger 2002c; Ergül et al. 2005a; Demirel et al. 2006; Baba et al. 2013b), A. versicolor W. Phillips (Härkönen 1988; Ergül \& Dülger 2000d; Ocak \& Hasenekoğlu 2003a, 2005; Oran et al. 2006; Ergül \& Akgül 2011; Baba et al. 2013b, 2015)

\section{Dianemataceae}

Calomyxa metallica (Berk.) Nieuwl. (Ergül \& Dülger 2000d; Oran et al. 2006)

Dianema corticatum Lister (Ocak \& Hasenekoğlu 2005), D. harveyi Rex (Ergül et al. 2005b), D. repens G. Lister \& Cran (Ergül \& Dülger 2002c; Ergül \& Akgül 2011)

\section{Trichiaceae}

Arcyodes incarnata (Alb. \& Scwein.) O.F. Cook (Yağız \& Afyon 2006a, c, 2007a; Eroğlu \& Kaşik 2013a; Yildiz \& Dülger 2015)

Hemitrichia abietina (Wigand) G. Lister (Baba \& Tamer 2008b; Baba et al. 2013b), H. calyculata (Speg.) M.L. Farr (Ergül \& Dülger 2000d, 2002a; Ergül et al. 2005a, b; Oran et al. 2006; Yağız \& Afyon 2007b), H. clavata (Pers.) Rostaf. (Härkönen 1988; Ergül \& Dülger 2000d; Yağı \& Afyon 2007b; Baba et al. 2013b), H. leiocarpa (Cooke) Lister (Oran \& Ergül 2004; 
Yağız \& Afyon 2007b), H. minor G. Lister (Oran \& Ergül 2004; Ergül et al. 2005b; Yağız \& Afyon 2007b)

Metatrichia floriformis (Schwein.) Nann.-Bremek. (Oran \& Ergül 2004), M. vesparia (Batsch) Nann.-Bremek. ex G.W. Martin \& Alexop. (Ergül \& Gücin 1993; Ergül \& Dülger 2000d; Ergül et al. 2005a; Yağız \& Afyon 2005, 2007b; Oran et al. 2006)

Oligonema flavidum (Peck) Peck (Ocak 2015), O. schweinitzii (Berk.) G.W. Martin (Ocak \& Hasenekoğlu 2003b; Baba et al. 2013b; Eroğlu \& Kaşik 2013a)

Perichaena chrysosperma (Curr.) Lister (Ergül \& Dülger 1998, 2000d; Ergül et al. 2005b; Ocak \& Hasenekoğlu 2005; Yağız \& Afyon 2005, 2006a; Oran et al. 2006; Baba \& Tamer 2008a; Eroğlu \& Kaşik 2013a), P. corticalis (Batsch) Rostaf. (Härkönen \& Uotila 1983; Härkönen 1988; Ergül \& Dülger 2000d; Ocak \& Hasenekoğlu 2003a, 2005; Ergül et al. 2005b; Oran et al. 2006; Baba \& Tamer 2008a; Baba et al. 2013b, 2015; Eroğlu \& Kaşik 2013a; Ocak 2015; Oskay \& Tüzün 2015), P. depressa Lib. (Yağız \& Afyon 2007b; Demirel et al. 2010; Baba 2012; Baba et al. 2013b, 2015; Eroğlu \& Kaşik 2013a; Baba \& Zümre 2015), P. tessellata G. Lister (Oran \& Ergül 2004; Ergül et al. 2005b), P. vermicularis (Schwein.) Rostaf. (Ergül \& Dülger 2000d; Ocak $\&$ Hasenekoğlu 2003a; Ergül et al. 2005b; Oran et al. 2006; Baba \& Tamer 2008a; Baba et al. 2013b, 2015)

Prototrichia metallica (Berk.) Massee (Baba 2008)

Trichia affinis de Bary (Ergül \& Dülger 2002c; Ergül et al. 2005a; Oran et al. 2006), T. alpina (R.E. Fr.) Meyl. (Härkönen 1988; Ergül \& Dülger 2000d), T. botrytis (J.F. Gmel.) Pers. (Ergül \& Dülger 2000d; Ocak \& Hasenekoğlu 2003a, 2005; Ergül et al. 2005a; Oran et al. 2006; Yağız \& Afyon 2006a; Baba \& Tamer 2008a; Ergül \& Akgül 2011; Baba et al. 2013b, 2015), T. contorta (Ditmar) Rostaf. (Härkönen \& Uotila 1983; Ergül \& Gücin 1995; Ergül \& Dülger 2000d; Ergül et al. 2005b; Demirel et al. 2006; Oran et al. 2006; Baba \& Tamer 2008a; Eroğlu \& Kaşik 2013a), T. crateriformis G.W. Martin (Baba \& Tamer 2008a; Ergül \& Akgül 2011), T. decipiens (Pers.) T. Macbr. (Härkönen 1988; Ergül \& Dülger 2000d; Yağız et al. 2002; Ocak \& Hasenekoğlu 2003a, 2005; Ergül et al. 2005a, b; Yağız \& Afyon 2005, 2006a, 2007b; Demirel et al. 2006; Oran et al. 2006; Baba \& Tamer 2008a; Ergül \& Akgül 2011; Baba et al. 2015; Ocak 2015; Oskay \& Tüzün 2015), T. erecta Rex (Ocak \& Hasenekoğlu 2005; Baba et al. 2013b; Baba \& Zümre 2015), T. favoginea (Batsch) Pers. (Härkönen 1988; Ergül \& Dülger 2000d; Ergül et al. 2005a; Ocak \& Hasenekoğlu 2005; Yağız \& Afyon 2005; Baba \& Tamer 2008a; Ergül \& Akgül 2011), T. flavicoma (Lister) Ing (Yağız \& Afyon 2006a), T. lutescens (Lister) Lister (Gücin \& Öner 1986; Ergül \& Dülger 2000d; Demirel et al. 2006; Baba \& Tamer 2008a; Baba et al. 2013b; Baba \& Zümre 2015), T. munda (Lister) Meyl. (Baba et al. 2013b), T. persimilis P. Karst. (Baba et al. 2015), T. subfusca Rex (Oran \& Ergül 2004), T. varia 
(Pers. ex J.F. Gmel.) Pers. (Gücin \& Öner 1986; Ergül \& Dülger 2000d; Ergül et al. 2005a, b; Yağız \& Afyon 2005; Demirel et al. 2006; Oran et al. 2006; Baba \& Tamer 2008a; Ergül \& Akgül 2011; Baba et al. 2013b, 2015; Eroğlu \& Kaşik 2013a), T. verrucosa Berk. (Oran et al. 2006; Baba \& Tamer 2008a; Baba et al. 2013b, 2015)

\section{A list of synonyms}

Amaurochaete fuliginosa (Sowerby) T. Macbr. = Amaurochaete atra

Arcyria gulielmae Nann.-Bremek. = Arcyria minuta; Arcyria nutans (Bull.) Grev. = Arcyria obvelata

Badhamia dearnessii Hagelst. = Badhamia goniospora; Badhamia gracilis (T. Macbr.) T. Macbr. = Badhamia melanospora; Badhamia nitens var. reticulata (Berk. \& Broome ex Massee) G. Lister = Badhamia nitens

Collarra elegans (Racib.) Dhillon \& Nann.-Bremek. ex Ing = Comatricha elegans

Comatricha acanthodes Alexop. = Paradiacheopsis acanthodes; Comatricha lurida Lister

= Collaria lurida; Comatricha pulchella var. fusca (Lister) G. Lister = Comatricha pulchella var. pulchella

Cribraria cancellata var. fusca (Lister) Nann.-Bremek = Cribraria cancellata var. cancellata Dictydium Schrad. = Cribraria; Dictydium cancellatum (Batsch) T. Macbr. = Cribraria cancellata

Enteridium liceoides (Lister) G. Lister = Reticularia liceoides; Enteridium splendens (Morgan)

T. Macbr. = Reticularia splendens; Enteridium splendens var. juranum (Meyl.) Härk. = Reticularia jurana

Famintzinia fruticulosa (O.F. Müll.) Lado = Ceratiomyxa fruticulosa

Fuligo candida Pers. = Fuligo septica

Hemitrichia imperialis G. Lister = Arcyria imperialis; Hemitrichia karstenii (Rostaf.) Lister

= Trichia contorta

Hyporhamma calyculatum (Speg.) Lado = Hemitrichia calyculata; Hyporhamma clavatum (Pers.) Lado = Hemitrichia clavata; Hyporhamma leiocarpum (Cooke) Lado (as $H$. leiocarpa) = Hemitrichia leiocarpa; Hyporhamma minus (G. Lister) Lado (as H. minor)

= Hemitrichia minor

Lachnobolus ater (Alb. \& Schwein.) Lado (as L. atrus) = Amaurochaete atra

Lamproderma atrosporum Meyl. = Meriderma cribrarioides

Lycogala terrestre Fr. = Lycogala epidendrum

Macbrideola cornea var. macrospora Nann.-Bremek. = Macbrideola macrospora

Physarum nutans Pers. = Physarum album

Stemonitis hyperopta Meyl. = Stemonitopsis hyperopta; Stemonitis nigrescens $\mathrm{Rex}=$ Stemonitis fusca

Stemonitopsis irregularis (Rex) T.N. Lakh. \& Mukerji = Stemonaria irregularis

Trichia contorta var. karstenii (Rostaf.) Ing = Trichia contorta var. contorta; Trichia decipiens var. olivacea (Meyl.) Meyl. = Trichia crateriformis

Tubulifera arachnoidea Jacq. = Tubifera ferruginosa 


\section{Excluded records}

Badhamia utricularis var. microspora Dülger \& Gönüz (Dülger \& Gönüz, 2005; as 'B. microsporus') (nom. inval., Art. 40.6)

Comatricha dictyospora L.F. Čelak. (nom. dub.; see Martin \& Alexopoulos 1969: 277; Lado 2016)

\section{Discussion}

The total number of correct names of species of myxomycetes, recognized as occurring in Turkey, is 252 (Table 1). Two taxa were treated as doubtfoul or had invalidly published names and thus were included in a list of excluded records, providing reasons for their exclusion.

Table 1. Diversity of myxomycetes in Turkey

\begin{tabular}{|c|c|c|}
\hline Orders/Families & Genera & Species \\
\hline \multicolumn{3}{|l|}{ Acrasida } \\
\hline Guttulinaceae & Pocheina & 1 \\
\hline \multicolumn{3}{|l|}{ Protostelida } \\
\hline Ceratiomyxaceae & Ceratiomyxa & 1 \\
\hline \multicolumn{3}{|l|}{ Echinostellida } \\
\hline Clastodermataceae & Clastoderma & 2 \\
\hline Echinosteliaceae & Echinostelium & 10 \\
\hline Liceida & Cribraria & 16 \\
\hline \multirow[t]{2}{*}{ Cribrariaceae } & Lindbladia & 1 \\
\hline & Dictydiaethalium & 1 \\
\hline Dictydiaethaliaceae & Licea & 21 \\
\hline Liceaceae & Lycogala & 3 \\
\hline \multirow[t]{4}{*}{ Tubiferaceae } & Reticularia & 4 \\
\hline & Tubifera & 1 \\
\hline & Diderma & 9 \\
\hline & Didymium & 21 \\
\hline Physarida & Mucilago & 1 \\
\hline \multirow[t]{3}{*}{ Didymiaceae } & Badhamia & 15 \\
\hline & Badhamiopsis & 1 \\
\hline & Craterium & 3 \\
\hline \multirow[t]{5}{*}{ Physaraceae } & Fuligo & 2 \\
\hline & Leocarpus & 1 \\
\hline & Physarum & 32 \\
\hline & Protophysarum & 1 \\
\hline & Willkommlangea & 1 \\
\hline
\end{tabular}


Table 1. (continued)

\begin{tabular}{|c|c|c|}
\hline Orders/Families & Genera & Species \\
\hline \multirow{14}{*}{$\begin{array}{l}\text { Stemonitida } \\
\text { Stemonitidaceae }\end{array}$} & Amaurochaete & 3 \\
\hline & Collaria & 3 \\
\hline & Comatricha & 11 \\
\hline & Diachea & 1 \\
\hline & Diacheopsis & 1 \\
\hline & Enerthenema & 1 \\
\hline & Lamproderma & 4 \\
\hline & Macbrideola & 7 \\
\hline & Meriderma & 1 \\
\hline & Paradiacheopsis & 6 \\
\hline & Stemonaria & 1 \\
\hline & Stemonitis & 11 \\
\hline & Stemonitopsis & 7 \\
\hline & Symphytocarpus & 4 \\
\hline \multirow{5}{*}{$\begin{array}{l}\text { Trichiida } \\
\qquad \begin{array}{l}\text { Arcyriaceae } \\
\text { Dianemataceae }\end{array}\end{array}$} & Arcyria & 19 \\
\hline & Calomyxa & 1 \\
\hline & Dianema & 3 \\
\hline & Arcyodes & 1 \\
\hline & Hemitrichia & 5 \\
\hline \multirow{5}{*}{ Trichiaceae } & Metatrichia & 2 \\
\hline & Oligonema & 2 \\
\hline & Perichaena & 5 \\
\hline & Prototrichia & 1 \\
\hline & Trichia & 15 \\
\hline Total & 46 & 252 \\
\hline
\end{tabular}

The richest genera in terms of the number of species are Physarum (32 species), Licea (21), Didymium (21) and Arcyria (19).

Acknowledgements. The authors are grateful to all mycologists and botanists who kindly provided them with copies of articles with records from Turkey.

\section{References}

Baba, H. 2008. A new Myxomycetes genus and three species record for Turkey. - International Journal of Botany 4: 336-339. http://dx.doi.org/10.3923/ijb.2008.336.339

Baba, H. 2012. Myxomycetes of Mustafa Kemal University Campus and environs (Turkey). - Turkish Journal of Botany 36: 769-777. http://dx.doi.org/10.3906/bot-1103-10 
Baba, H. 2015. The genus Physarum (Myxomycetes) checklist in Turkey. - Biological Diversity and Conservation 8(3): 20-24.

Baba, H. \& Gelen, M. 2014. Two new Myxomycetes (Physarum albescens Ellis ex T. Macbr. and Physarum tropicale T. Macbr.) record from Turkey. - Biological Diversity and Conservation 7(3): 190-194.

Baba, H. \& Tamer, A.Ü. 2008a. A study on the Myxomycetes in Manisa. - The Herb Journal of Systematic Botany 14(2): 179-196.

Baba, H. \& Tamer, A.Ü. 2008b. A new myxomycetes genus and three species record for Turkish mycoflora.The Herb Journal of Systematic Botany 15(2): 81-86.

Baba, H. \& Zümre, M. 2015. [Myxomycetes of Alan Plateau (Hatay)]. - The Journal of Fungus 6(1): 1-9. (In Turkish). http://dx.doi.org/10.15318/Fungus.2015610967

Baba, H., Tamer, A.Ü. \& Kalyoncu, F. 2008. New myxomycete records for Turkey: one new genus and three new species. - Turkish Journal of Botany 32: 329-332.

Baba, H., Gelen, M. \& Zümre, M. 2012. A new Physarum (Myxomycetes) record from Hatay-Turkey. - The Herb Journal of Sytematic Botany 19(2): 125-131.

Baba, H., Gelen, M. \& Zümre, M. 2013a. A new Myxomycetes record for Physarum genus from Turkey. Biological Diversity and Conservation 6(3): 49-51.

Baba, H., Zümre, M. \& Gelen, M. 2013b. Biodiversity of Kuseyr Plateau Myxomycetes (Hatay-Turkey).Journal of Selcuk University, Natural and Applied Science, Special Issue, ICOEST Conf. 2013 (Digital Proceeding of the International Conference on Environmental Science and Technology - 2013, Cappadocia, Turkey, 18-21 June 2013) (Part 1): 669-683.

Baba, H., Gelen, M. \& Zümre, M. 2014. [First report of fimicolous myxomycetes from Turkey]. - The Journal of Fungus 5(1): 1-6. (In Turkish). http://dx.doi.org/10.15318/Fungus.201456195

Baba, H., Kolukırık, M. \& Zümre, M. 2015. Differentiation of some myxomycetes species by ITS sequences. - Turkish Journal of Botany 39: 377-382. http://dx.doi.org/10.3906/bot-1405-12

Davis, P.H. 1965-1988. Flora of Turkey and the East Aegean Islands. Edinburgh University Press, Edinburgh.

Davis, P.H., Happer, P.C. \& Hedge, I.C. (eds) 1971. Plant life of South-West Asia. The Botanical Society of Edinburgh, Edinburgh.

Demirel, G. \& Kaşık, G. 2012. Four new records for Physarales from Turkey. - Turkish Journal of Botany 36: 95-100. http://dx.doi.org/10.3906/bot-1010-12

Demirel, K. \& Uzun, Y. 2002. Macrofungi of Ağr1 Province. - Turkish Journal of Botany 26: 291-295.

Demirel, G., Kaşık, G. \& Öztürk, C. 2006. Myxomycetes of Kestel Forest (Kadınhanı, Konya). - Turkish Journal of Botany 30: 441-447.

Demirel, G., Kaşık, G. \& Öztürk, C. 2010. [Contributions to Turkish Myxomycetes in Kestel Forests (Konya) . - The Journal of Fungus 1(1): 21-25. (In Turkish)

Demirel, K. \& Uzun, Y. 2002. Macrofungi of Ağr1 Province. - Turkish Journal of Botany 26: 291-295.

Demirel, K., Uzun, Y. \& Biber, G. 2004. Macrofungi of Şavşat (Artvin) District. - The Herb Journal of Sytematic Botany 11(2): 191-206.

Doğan, H.H. \& Eroğlu, G. 2014. A new Stemonitis species from Turkey. - Mycotaxon 129: 293-296. http://dx.doi.org/10.5248/129.293

Dülger, B. 2007. Checklist of the myxomycetes in Turkey. - Mycologia Balcanica 4: 151-155. 
Dülger, B. 2008a. Physarum galbeum (Physaraceae) in Turkey. - In: C.M. Denchev (ed.). New records of fungi, fungus-like organisms, and slime moulds from Europe and Asia: 1-6. - Mycologia Balcanica 5: 93-94.

Dülger, B. 2008b. Two new Myxomycetes records for the myxobiota of Turkey. - Turkish Journal of Botany 32: 333-335.

Dülger, B. 2008c. A new myxomycete record for Turkish myxobiota: Badhamia dubia (Physaraceae). - In: C.M. Denchev (ed.). New records of fungi, fungus-like organisms, and slime moulds from Europe and Asia: 7-13. - Mycologia Balcanica 5: 157.

Dülger, B. \& Duman, S. 2010. Physarum perfectum (Physaraceae) - a new myxomycete record for the myxobiota of Turkey - In: C.M. Denchev (ed.). New records of fungi, fungus-like organisms, and slime moulds from Europe and Asia: 20-27. - Mycologia Balcanica 7: 117-123.

Dülger, B. \& Gönüz, A. 2005. A new variety of Badhamia (Myxomycetes) from Turkey. - International Journal of Botany 1(1): 90-92. http://dx.doi.org/10.3923/ijb.2005.90.92

Dülger, B., Karabacak, E., Süerdem, T.B. \& Hacıŏlu, N. 2005. A new myxomycete record for the fungi flora of Turkey. - International Journal of Botany 1(1): 62-63. http://dx.doi.org/10.3923/ijb.2005.62.63

Dülger, B., Ergül, C.C., Süerdem, T.B. \& Oran, R.B. 2006. [The myxomycetes of Bozcaada (Çanakkale)]. The Herb Journal of Sytematic Botany 13(2): 189-194. (In Turkish)

Dülger, B., Süerdem, T.B. \& Hacioglu, N. 2007. A new myxomycete record for Turkish myxobiota: Comatricha suksdorfi. - Mycologia Balcanica 4: 77-78.

Ergül, C.C. 1992. Two new myxomycetes taxa for Turkish mycoflora. - IUFS Journal of Biology 56: 57-61. Ergül, C.C. \& Akgül, H. 2011. Myxomycete diversity of Uludağ National Park, Turkey. - Mycotaxon 116: 479 (a full checklist at http://www.mycotaxon.com/resources/checklists/ErgulAkgul-v116-checklist. pdf).

Ergül, C.C. \& Dülger, B. 1998. The myxomycetes of Görükle (Bursa) campus area. - The Herb Journal of Systematic Botany 5(1): 93-96.

Ergül, C.C. \& Dülger, B. 1999. [A new myxomycetes taxon for the Turkish mycoflora: Symphytocarpus flaccidus Ing \& Nann.-Brem.]. - The Herb Journal of Systematic Botany 6(1): 99-102. (In Turkish)

Ergül, C.C. \& Dülger, B. 2000a. Three new records of Paradiacheopsis Hertel for the Turkish myxomycetes flora. - In: N. Özhatay (ed.). Second Balkan Botanical Congress, İstanbul, 14-18 May 2000. Vol. 1. Pp. 201-206. İstanbul University, İstanbul.

Ergül, C. \& Dülger, B. 2000b. A new myxomycetes record for the Turkish mycoflora. - Turkish Journal of Botany 24: 289-291.

Ergül, C.C. \& Dülger, B. 2000c. A new myxomycetes genus record for Turkey (Stemonitopsis (Nann.-Brem.) Nann.-Brem.). - Turkish Journal of Botany 24: 355-357.

Ergül, C.C. \& Dülger, B. 2000d. Myxomycetes of Turkey. - Karstenia 40: 39-41.

Ergül, C. \& Dülger, B. 2002a. Two new records of myxomycete taxa for Turkish mycoflora. -The Herb Journal of Systematic Botany 9(1): 129-136.

Ergül, C.C. \& Dülger, B. 2002b. A new record for the myxomycetes flora of Turkey: Comatricha pulchella (C. Bab.) Rost. var. pulchella. - Turkish Journal of Botany 26: 113-115.

Ergül, C.C. \& Dülger, B. 2002c. New records for the myxomycetes flora of Turkey. - Turkish Journal of Botany 26: 277-280.

Ergül, C.C. \& Gücin, F. 1993. [Two new myxomycetes taxa for Turkey]. - Turkish Journal of Botany 17: 267-271. (In Turkish) 
Ergül, C.C. \& Gücin, F. 1994. [A new record for Turkish myxomycetes: (Fuligo septica (L.) Wiggers)]. In: N. Aktaç et al. (eds). XII. Ulusal Biyoloji Kongresi, Edirne, 6-8 July 1994. Pp. 157-159. Trakya Üniversitesi, Edirne. (In Turkish)

Ergül, C.C. \& Gücin, F. 1995. [A new myxomycetes taxon for Turkey: Hemitrichia Rost.]. - Turkish Journal of Botany 19: 165-166. (In Turkish)

Ergül, C.C. \& Oran, R.B. 2005. Three new records for the Turkish myxobiota. - Turkish Journal of Botany 29: 241-242.

Ergül, C.C., Dülger, B. \& Akgül, H. 2005a. Myxomycetes of Mezit Stream valley of Turkey. - Mycotaxon 92: 239-242 (a full checklist at http://biyoloji.uludag.edu.tr /ergul/ Checklist_001.pdf).

Ergül, C.C., Dülger, B., Oran, R.B. \& Akgül, H. 2005b. Myxomycetes of the western Black Sea Region of Turkey. - Mycotaxon 93: 269-272 + 1-9 (a full checklist at http://biyoloji.uludag.edu.tr/ergul/ Checklist_002.pdf).

Eroğlu, G. \& Kaşik, G. 2013a. Myxomycete of Hadim and Taşkent districts (Konya/Turkey) and their ecology. - Biological Diversity and Conservation 6(3): 120-127.

Eroğlu, G. \& Kaşik, G. 2013b. Six new myxomycete records from Turkey. - Journal of Applied Biological Sciences 7(1): 75-78.

Eroğlu, G., Öztürk, C., Kaşik, G., Doğan, H.H., Aktaş, S. \& Alkan, S. 2014. Some myxomycete from Çivril (Denizli-Turkey) province. - Journal of Selçuk University, Natural and Applied Science 3(4): 21-26. Eroğlu, G., Kaşik, G. \& Öztürk, C. 2015. Three new myxomycete records from Turkey. - Biological Diversity and Conservation 8(1): 16-18.

Gücin, F. \& Ergül, C. 1995. A new myxomycete genus (Enteridium) record for the Turkish mycoflora. Turkish Journal of Botany 19: 565-566.

Gücin, F. \& Öner, M. 1986. Taxonomic observations on some Turkish Myxomycetes species. - Journal of Firat University 1(1): 19-28.

Gücin, F., Gün, Z., Ergül, C.C. \& Dülger, B. 1996a. A new record of Subdivison Acrasiogymnomycotina for Turkish mycoflora, Pocheina rosea (Cienk.) Loeblich et Tappan. - In: M.A. Öztürk, Ö. Seçmen \& G. Görk (eds). Plant Life in Southwest and Central Asia Symposium, İzmır, 21-28 May 1995. Vol. 1. Pp. 427-431. Ege University Press, İzmır, Turkey.

Gücin, F., Solak, M.H. \& Işsloğlu, M. 1996b. Mushrooms of Uludağ (Bursa-Turkey). - In: M. Öztürk, Ö. Seçmen \& G. Görk (eds). Plant Life in Southwest and Central Asia Symposium, İzmır, 21-28 May 1995. Vol. 1. Pp. 402-413. Ege University Press, İzmır, Turkey.

Gün, Z., Gücin, F. \& Ergül, C.C. 1996. [The myxomycetes taxa determined from Uludağ vegetation zone]. - In: A. Özalpan (ed.). XIII. Ulusal Biyoloji Kongresi, İstanbul, 17-20 September 1996. P. 76. İstanbul Üniversitesi, İstanbul. (In Turkish)

Härkönen, M. 1988. Some additions to the knowledge of Turkish myxomycetes. - Karstenia 27[1987]: 1-7. Härkönen, M. \& Uotila, P. 1983. Turkish myxomycetes developed in moist chamber cultures. - Karstenia 23: 1-9.

Kaya, A. \& Demirel, K. 1998. Two new myxomycetes for the mycoflora of Turkey. - Bulletin of Pure and Applied Sciences 17B(2): 47-48.

Lado, C. 2016. An on line nomenclatural information system of Eumycetozoa. Real Jardín Botánico, CSIC. Madrid, Spain. http://www.nomen.eumycetozoa.com.

Lohwag, K. 1957. [Research on Turkish mycoflora]. - İstanbul Üniversitesi Orman Fakültesi Dergisi 7(1): 129-137. (In Turkish) 
Lohwag, K. 1964. Mykologische Notizen aus dem Belgrader Wald bei Istanbul in der Türkei. - Sydowia 16: 199-204.

Martin, G.W. \& Alexopoulos, C.J. 1969. The Myxomycetes. Univ. Iowa Press. Iowa.

Ministry of Environment and Forestry 2007. The National Biological Diversity Strategy and Action Plan 2007. Republic of Turkey, Ministry of Environment and Forestry, General Directorate of Nature Conservation and National Parks, Department of Nature Conservation, National Focal Point of Convention on Biological Diversity, Ankara.

Ocak, İ. 2015. Seasonal distribution of field-collected myxomycete in the Koroglubeli Forest, Afyonkarahisar, Turkey. - Ekoloji 24(96) 48-56. http://dx.doi.org/10.5053/ekoloji.2015.18

Ocak, İ. \& Hasenekoğlu, İ. 2003a. Myxomycetes from Erzurum, Bayburt and Gümüşhane provinces (Turkey). - Turkish Journal of Botany 27: 223-226.

Ocak, İ. \& Hasenekoğlu, İ. 2003b. Four new records of myxomycetes from Turkey. - Turkish Journal of Botany 27: 333-337.

Ocak, İ. \& Hasenekoğlu, İ. 2005. Myxomycetes from Trabzon and Giresun Provinces (Turkey). - Turkish Journal of Botany 29: 11-21.

Oran, R.B. \& Ergül, C.C. 2004. New records for the myxobiota of Turkey. - Turkish Journal of Botany 28: 511-515.

Oran, R.B. \& Ergül, C.C. 2015. New records of corticolous myxomycetes from Turkey. - Turkish Journal of Botany 130: 181-190. http://dx.doi.org/10.5248/130.181

Oran, R.B., Ergül, C.C. \& Dülger, B. 2006. Myxomycetes of Belgrad Forest (Istanbul). - Mycotaxon 97: 183-187. (a full checklist at http://biyoloji.uludag.edu.tr/ergul/checklist_003.pdf)

Oskay, M. \& Tüzün, Ö. 2015. [Determination the myxobiota of Kemalpaşa and surrounding (İzmir)]. Celal Bayar University Journal of Science 11(1): 59-68. (In Turkish)

Pekşen, A. \& Karaca, G.H. 2000. [Macrofungi of Haciosman forest (Samsun)]. - The Herb Journal of Systematic Botany 7(1): 211-218. (In Turkish)

Pekşen, A. \& Karaca, G.H. 2003. Macrofungi of Samsun Province. - Turkish Journal of Botany 27: 173-184.

Selik, M. 1964. [Mycological notes from Belgrad forest]. - İstanbul Üniversitesi Orman Fakültesi Dergisi 14(2): 129-135. (In Turkish)

Sesli, E. \& Denchev, C.M. 2005. Checklists of the myxomycetes and macromycetes in Turkey. - Mycologia Balcanica 2: 119-160.

Sesli, E. \& Denchev, C.M. 2008. Checklists of the myxomycetes, larger ascomycetes, and larger basidiomycetes in Turkey. - Mycotaxon 106: 65-67.

Sesli, E. \& Denchev, C.M. 2014. Checklists of the myxomycetes, larger ascomycetes, and larger basidiomycetes in Turkey. $6^{\text {th }}$ edn. - Mycotaxon Checklists Online (http://www.mycotaxon.com/ resources/checklists/sesli-v106-checklist.pdf): 1-136.

Sümer, S. 1982. [Wood-decaying fungi in the western Black Sea Region of Turkey, especially in and around Bolu Province]. - İstanbul Üniversitesi Orman Fakültesi Yayınları 312: 1-94. (In Turkish)

Türkekul, İ. 2003. A contribution to the fungal flora of Tokat Province. - Turkish Journal of Botany 27: 313-320.

Yağız, D. \& Afyon, A. 2003. [Two new records for the Turkish myxomycete flora]. - Selçuk Üniversitesi Eğitim Fakültesi Fen Bilimleri Dergisi 15: 563-568. (In Turkish)

Yağız, D. \& Afyon, A. 2005. [A study on the myxomycetes of Seydişehir (Konya) District]. - Afyon Kocatepe Üniversitesi Fen Bilimleri Dergisi 5(1): 55-60. (In Turkish) 
Yağız, D. \& Afyon, A. 2006a. Myxomycete flora of Derebucak (Konya) and Akseki (Antalya) districts in Turkey. - Mycotaxon 96: 257-260 + 1-16 (a checklist at http://www.egitim.selcuk.edu.tr/fen/yagiz/ pdf/mycotaxon06-092.pdf).

Yağız, D. \& Afyon, A. 2006b. [Two new records for Turkish myxomycetes]. - The Herb Journal of Sytematic Botany 13(1): 23-26. (In Turkish)

Yağız, D. \& Afyon, A. 2006c. [Four new records for myxomycetes flora in Turkey]. - In: K. Olgun (ed.). 18. Ulusal Biyoloji Kongresi, Kuşadası, 26-30 June 2006. Pp. 219-220. Adnan Menderes Üniversitesi, Aydın. (In Turkish)

Yağız, D. \& Afyon, A. 2007a. Three new records for myxomycetes of Turkey. - Turkish Journal of Botany 31: 467-470.

Yağız, D. \& Afyon, A. 2007b. The ecology and chorology of myxomycetes in Turkey. - Checklist to Mycotaxon 101: 279-282 + 1-19 (a checklist at http://egitim.selcuk.edu.tr/fen/yagiz/pdf/ mycotaxon07-080.pdf).

Yağız, D., Ergül, C.C. \& Afyon, A. 2002. [A study on the myxomycetes in Beyşehir (Konya)]. - The Herb Journal of Systematic Botany 9(1): 137-141. (In Turkish)

Yildiz, İ. \& Dülger, B. 2015. [The first fungicolous myxomycete record from Turkey]. - Düzce Üniversitesi Bilim ve Teknoloji Dergisi 3: 350-356. (In Turkish)

Zümre, M., Baba, H. \& Gelen, M. 2013. [A new myxomycetes variety record from Turkey]. - The Black Sea Journal of Sciences 3(8): 47-51. (In Turkish) 